\title{
A Class of Inhomogeneous Cosmological Models
}

\author{
P. Szekeres \\ Department of Mathematical Physics, University of Adelaide, Adelaide, Australia
}

Received March 21, 1974

\begin{abstract}
All solutions of Einstein's field equations representing irrotational dust and possessing a metric of the form $d s^{2}=d t^{2}-e^{2 \alpha} d r^{2}-e^{2 \beta}\left(d y^{2}+d z^{2}\right)$ are found. The new metrics generalize the earlier Bondi-Tolman, Eardley-Liang-Sachs, and Kantowski-Sachs cosmological models.
\end{abstract}

\section{Introduction}

Cosmological solutions to Einstein's field equations are generally found [1] by imposing a large group symmetries on the metric, amounting to an assumption of spatial homogeneity. While this assumption is probably quite reasonable in an averaged sense, it is obviously false on galactic and smaller scales. It is therefore of considerable interest to have at hand a large variety of inhomogeneous models as a basis of comparison with the homogeneous ones. All questions of detail such as galaxy formation or the detailed structure of the black-body radiation, to be treated properly, should ultimately be referred to such inhomogeneous models. In particular the study of the singularity structure of more general models is still only in its initial stages [2], and promises to lead to radical departures from the highly idealized Friedmann-type singularities which are usually considered. However the only generally known inhomogeneous solutions are the spherically symmetric Bondi-Tolman metrics [3] and the plane-symmetric models of EardleyLiang and Sachs [2] (referred to as ELS in this paper), and both of these examples still impose strong symmetry groups.

In this paper we abandon all a priori symmetry assumptions, but concentrate our attention on metrics having the simple form

$$
d s^{2}=d t^{2}-e^{2 \alpha} d r^{2}-e^{2 \beta}\left(d y^{2}+d z^{2}\right) .
$$

All solutions of this type will be found which represent irrotational dust,

$$
G_{\mu \nu}=\kappa T_{\mu \nu}=\kappa \varrho u_{\mu} u_{v}
$$

where $u_{\mu}=(1,0,0,0), \kappa=8 \pi G(c=1)$. Our solutions will be found to generalize both the Bondi-Tolman and ELS solutions, by essentially displacing the "centres of symmetry" in a well-determined manner, leading to what might be termed quasi-spherical and quasi-planar metrics (as well as a new class of quasi-pseudospherical metrics which generalize a previously unexplored class of pseudospherically symmetric metrics). A second class of solutions is also found which generalizes the homogeneous cylindrical solutions of Kantowski-Sachs [4] in an analogous fashion. Wheter this displacement process is part of a more 
general procedure for obtaining new solutions from old ones is not at present clear, but the solutions presented here are strongly suggestive of the existence of a more general result of this type.

\section{Friedmann Functions}

Consider a metric of the form

$$
d s^{2}=d t^{2}-\bar{g}_{a b} d x^{a} d x^{b} \quad(a, b=1,2,3) .
$$

The Einstein field equations (2) for irrotational dust read

where

$$
\begin{aligned}
& G_{0}^{0}=-\bar{G}-\frac{1}{2} K_{a}^{b} K_{b}^{a}+\frac{1}{2} K^{2}=\kappa \varrho, \\
& G_{b}^{0}=K_{b \mid a}^{a}-K_{, b}=0, \\
& G_{b}^{a}=-\bar{G}_{b}^{a}-\dot{K}_{b}^{a}-K K_{b}^{a}+\delta_{b}^{a}\left(\dot{K}+\frac{1}{2} K^{2}+\frac{1}{2} K_{d}^{c} K_{c}^{d}\right)=0,
\end{aligned}
$$

$$
K_{a b}=\frac{1}{2} \dot{\bar{g}}_{a b}, K_{a}^{b}=\bar{g}^{b c} K_{a c}, K=K_{a}^{a}, \cdot \equiv \partial / \partial t,
$$

| refers to covariant derivative taken with respect to the 3-metric $\bar{g}_{a b}, \bar{G}_{b}^{a}$ is the Einstein tensor of the 3-metric, and $\bar{G}=\bar{G}_{a}^{a}$.

As an alternative to Eq. (3) we obtain from (3) and (广)

$$
\dot{K}+K_{b}^{a} K_{a}^{b}+\frac{1}{2} \kappa \varrho=0,
$$

Raychaudhuri's Eq. [5], which may be used to show that along each streamline the density becomes infinite at some finite time $t_{0}$.

Now if $\bar{g}_{a b}=e^{2 \psi} \hat{g}_{a b}$, where the $\hat{g}_{a b}$ are functions only of $x^{1}, x^{2}, x^{3}$, then we may show that the metric is a Friedmann model, for in this case

and substitution in (4) gives

$$
K_{b}^{a}=\dot{\psi} \delta_{b}^{a}
$$

$$
\dot{\psi}_{b}=0,
$$

whence $\psi=f(t)+h\left(x^{1}, x^{2}, x^{3}\right)$. It is clearly possible to absorb the function $h$ into the definition of $\hat{g}_{a b}$, so without loss of generality we may assume $\psi=\psi(t)$. Substituting into Eq. (5) results in

Thus

$$
\hat{G}_{b}^{a}\left(x^{1}, x^{2}, x^{3}\right)=e^{2 \psi} \bar{G}_{b}^{a}=\delta_{b}^{a} e^{2 \psi}\left(2 \ddot{\psi}+3 \dot{\psi}^{2}\right) .
$$

and

$$
e^{2 \psi}\left(2 \ddot{\psi}+3 \dot{\psi}^{2}\right)=-k, \quad k=\text { const. }
$$

$$
\hat{G}_{b}^{a}=-k \delta_{b}^{a} \text {. }
$$

Hence the 3-space defined by $\hat{g}_{a b}$ is a space of constant curvature and the metric may be brought to the Robertson-Walker form, giving rise to the Friedmann models.

Let us write $\psi=\log \phi$, so that (7) reads

$$
2 \ddot{\phi} \phi+\dot{\phi}^{2}=-k \text {, }
$$

and has a first integral

$$
\dot{\phi}^{2}=-k+\frac{l}{\phi}, \quad l=\text { const. }
$$


The general solution of this equation is

$$
\phi=F\left(k, l ; t-t_{0}\right), \quad t_{0}=\text { const. }
$$

where the Friedmann functions $F(k, l ; t)$ are defined as follows:

(i) $k>0, l>0 ; F(k, l ; t)=l k^{-1} \cos ^{2} \eta, t=l k^{-3 / 2}\left(\eta+\frac{1}{2} \sin 2 \eta\right)$,

(iia) $k<0, l<0 ; F(k, l ; t)=l k^{-1} \cosh ^{2} \eta, t=-l(-k)^{-3 / 2}\left(\eta+\frac{1}{2} \sinh 2 \eta\right)$,

(iib) $k<0, l>0 ; F(k, l ; t)=-l k^{-1} \sinh ^{2} \eta, t=l(-k)^{-3 / 2}\left(-\eta+\frac{1}{2} \sinh 2 \eta\right)$,

(iic) $k<0, l=0 ; F(k, 0 ; t)=(-k)^{1 / 2} t$,

(iiia) $k=0, l>0 ; F(0, l ; t)=(9 l / 4)^{1 / 3} t^{2 / 3}$,

(iiib) $k=0, l=0 ; F(0,0 ; t)=$ const.

These functions are defined as solutions of (8) which vanish at $t=0$ [except in the trivial case (iiib)], and which are positive for some interval $t>0$. This clearly eliminates from consideration the cases $k>0, l \leqq 0$ and $k=0, l<0$.

\section{Field Equations}

Returning to the metric (1), let us introduce a pair of complex variables,

$$
\xi=y+i z, \quad \bar{\xi}=y-i z .
$$

The field equations (4) and (5) reduce to

$$
\begin{aligned}
\frac{1}{2} G_{1}^{0} & =-\dot{\beta}^{\prime}+\beta^{\prime}(\dot{\alpha}-\dot{\beta})=0, \\
G_{\xi}^{0} & =-\dot{\alpha}_{\xi}-\dot{\beta}_{\xi}+\alpha_{\xi}(\dot{\beta}-\dot{\alpha})=0, \\
G_{\bar{\xi}}^{0} & =-\dot{\alpha}_{\bar{\xi}}-\dot{\beta}_{\bar{\xi}}+\alpha_{\bar{\xi}}(\dot{\beta}-\dot{\alpha})=0, \\
e^{2 \alpha} G_{\xi}^{1} & =\beta_{\xi}^{\prime}-\beta^{\prime} \alpha_{\xi}=0, \\
e^{2 \alpha} G_{\xi}^{1} & =\beta_{\bar{\xi}}^{\prime}-\beta^{\prime} \alpha_{\bar{\xi}}=0, \\
\frac{1}{2} e^{2 \beta} G_{\xi}^{\bar{\xi}} & =\alpha_{\xi \xi}+\left(\alpha_{\xi}\right)^{2}-2 \beta_{\xi} \alpha_{\xi}=0, \\
\frac{1}{2} e^{2 \beta} G_{\bar{\xi}}{ }^{\xi} & =\alpha_{\bar{\xi} \bar{\xi}}+\left(\alpha_{\bar{\xi}}\right)^{2}-2 \beta_{\bar{\xi}} \alpha_{\bar{\xi}}=0, \\
G_{1}{ }^{1} & =-4 e^{-2 \beta} \beta_{\xi \bar{\xi}}-e^{-2 \alpha}\left(\beta^{\prime}\right)^{2}+2 \ddot{\beta}+3 \dot{\beta}^{2}=0, \\
G_{\xi}{ }^{\xi}=G_{\bar{\xi}}{ }^{\bar{\xi}} & =e^{-2 \alpha}\left(-\beta^{\prime \prime}+\alpha^{\prime} \beta^{\prime}-\left(\beta^{\prime}\right)^{2}\right)-2 e^{-2 \beta}\left(\alpha_{\xi \bar{\xi}}+\alpha_{\xi} \alpha_{\bar{\xi}}\right)+\ddot{\alpha}+\ddot{\beta}+\dot{\alpha}^{2} \\
& \quad+\dot{\beta}^{2}+\dot{\alpha} \dot{\beta}=0,
\end{aligned}
$$

where $^{\prime} \equiv \partial / \partial r$, and

$$
\begin{aligned}
& \alpha_{\xi} \equiv \partial \alpha / \partial \xi=\frac{1}{2}(\partial \alpha / \partial y-i \partial \alpha / \partial z) \\
& \alpha_{\bar{\xi}} \equiv \partial \alpha / \partial \bar{\xi}=\frac{1}{2}(\partial \alpha / \partial y+i \partial \alpha / \partial z)
\end{aligned}
$$

The density is determined from Eq. (6),

$$
\kappa \varrho=-2\left(\ddot{\alpha}+2 \ddot{\beta}+\dot{\alpha}^{2}+2 \dot{\beta}^{2}\right) .
$$


We note some immediate integrability conditions for Eqs. (10)-(15). Forming $\partial / \partial \bar{\xi}$ of (11) and $\partial / \partial \xi$ of $\left(11^{\prime}\right)$ and subtracting we arrive at

and

$$
\begin{aligned}
& \alpha_{\xi} \dot{\alpha}_{\bar{\xi}}=\alpha_{\bar{\xi}} \dot{\alpha}_{\xi} \\
& \alpha_{\xi} \dot{\beta}_{\bar{\xi}}=\alpha_{\xi} \dot{\beta}_{\xi} .
\end{aligned}
$$

From $\partial / \partial t$ of (13) and $\partial / \partial \xi$ of (11) we have

$$
\dot{\beta}_{\xi \xi}-2 \beta_{\xi} \dot{\beta}_{\xi}+2 \dot{\beta}_{\xi} \alpha_{\xi}=0
$$

and the complex conjugate of this equation results from $\left(13^{\prime}\right)$ and $\left(11^{\prime}\right)$. Finally, from $\partial / \partial \xi$ of $(10)$ and $\partial / \partial t$ of (12) we find

and its complex conjugate

$$
\beta^{\prime} \dot{\beta}_{\xi}=0
$$

$$
\beta^{\prime} \dot{\beta}_{\bar{\xi}}=0 \text {. }
$$

Thus when $\beta^{\prime} \neq 0$, we must have $\dot{\beta}_{\xi}=\dot{\beta}_{\bar{\xi}}=0$, or

$$
\beta=\psi(t, r)=v(r, \xi, \bar{\xi}) \text {. }
$$

In the next two sections we shall obtain all solutions of Eqs. (10) - (15) subject to the condition (20). As we shall see in Section 6, even in the case where $\beta^{\prime}=0$, this condition will turn out to be sufficiently general to include all solutions of our equations.

\section{The Case $\boldsymbol{\beta}^{\prime} \neq 0$}

As discussed above, we may assume in this case that $\beta$ has the form (20). Of course $\psi$ and $v$ are not uniquely determined by this equation, since it is possible to replace them by

$$
\psi+f(r) \text { and } v-f(r)
$$

for any arbitrary function $f(r)$. However from Eq. (10) it follows that $f(r)$ may be chosen such that

$$
\alpha=\log \left(\psi^{\prime}+v^{\prime}\right)+\psi .
$$

Eqs. (11), $\left(11^{\prime}\right),(12)$, and $\left(12^{\prime}\right)$ are now all automatically satisfied, and Eq. (14) imples that

$$
4 e^{-2 v} v_{\xi \xi}+1=e^{2 \psi}\left(2 \ddot{\psi}+3 \psi^{2}\right)=-k(r),
$$

so that for each value of $r$, the function $\psi(t, r)$ satisfies the Friedmann equation (7), and the 2-metric $e^{2 v} d \xi d \bar{\xi}$ is a surface of constant curvature $1+k(r)$. As shown in the Appendix, coordinates $\xi, \bar{\xi}$ may be chosen such that

where

$$
\begin{gathered}
e^{-v}=a(r) \xi \bar{\xi}+B(r) \xi+\bar{B}(r) \bar{\xi}+c(r), \\
a c-B \bar{B}=\frac{1}{4}(1+k(r)) .
\end{gathered}
$$

Eqs. (13), (13'), and (15) are now satisfied automatically, and putting $\psi=\log \phi$ we have the complete solution expressed in terms of the Friedmann functions introduced in Section 2,

$$
\phi=F\left(k(r), l(r) ; t-t_{0}(r)\right)
$$


where $l(r), t_{0}(r)$ are arbitrary functions of $r$. Substituting in the density Eq. (16) gives

$$
\kappa \varrho=e^{-3 \psi}\left(l^{\prime}+3 l v^{\prime}\right) /\left(\psi^{\prime}+v^{\prime}\right) .
$$

From (21) we have $\alpha=\alpha(r, t)$ iff $v^{\prime}=v^{\prime}(r)$, i.e. iff there is a function $f(r)$ such that

$$
a=e^{f(r)} a_{1}, \quad B=e^{f(r)} B_{1}, \quad c=e^{f(r)} c_{1},
$$

where $a_{1}, B_{1}$, and $c_{1}$ are constants. It is clearly possible to pick a normalization such that

$$
K_{1}=4\left(a_{1} c_{1}-B_{1} \bar{B}_{1}\right)= \pm 1 \text { or } 0,
$$

and, as shown in the Appendix, coordinates $\xi, \bar{\xi}$ may be found such that $B_{1}=0$, $c_{1}=1 / 2$ and $a_{1}=K_{1} / 2$ whence

$$
1+k(r)=e^{2 f(r)} K_{1} .
$$

If $k(r)>-1$, the metric is spherically symmetric and reduces to a Tolman-Bondi model [3], if $k(r)=-1$ we have the class of plane symmetric models discussed by Eardley, Liang and Sachs [2], and $k(r)<-1$ is a class of "pseudo-spherically symmetric" models (i.e. a 3-parameter group of symmetries exists whose surfaces of transitivity are pseudospheres).

All vacuum solutions with $\beta^{\prime} \neq 0$ fall in the above category, for putting $\varrho=0$ in Eq. (26) implies

$$
l=0 \quad \text { or } \quad v=-\frac{1}{3} \log l(r)+v_{0}(\xi, \bar{\xi}) .
$$

The case $l=0$ gives flat space since a detailed computation shows that the Riemann tensor $R_{\mu \nu \varrho \sigma}$ vanishes. The second case is as above with $e^{f(r)}=(l(r))^{\frac{1}{3}}$, and reduces for $k(r)>-1$ to the Schwarzschild solution in comoving coordinates (see e.g. [6]), and for $k(r)<-1$ to its pseudo-spherically symmetric relative, which can be arrived at from the usual Schwarzschild coordinates by the complex substitution $\theta \rightarrow i \theta, d s^{2} \rightarrow-d s^{2}$, while the case $k(r)=-1$ can be obtained from Schwarzschild by a limiting procedure [7].

When the ratios $a: c: B$ are non-constant we have a generalization of the Bondi-Tolman, ELS and pseudo-spherically symmetric metrics to spaces with a set of displced or "non-concentric" spheres, planes and pseudospheres. We write out the complete metric explicitly in the case $k=0$ :

$$
d s^{2}=d t^{2}-\left(t-t_{0}\right)^{-2 / 3}\left(t-t_{1}\right)^{2} R^{2}(\partial \log P / \partial r)^{2} d r^{2}-\left(t-t_{0}\right)^{4 / 3} P^{-2} d \xi d \bar{\xi}
$$

where

$$
\begin{gathered}
\left.P=R(r)^{-1}[a(\xi+B / a)(\bar{\xi}+\bar{B} / a)+1 / 4 a)\right], \\
a=a(r), \quad B=B(r), \quad t_{0}=t_{0}(r), \quad R(r)=\left(\frac{9}{4} l(r)\right)^{1 / 3},
\end{gathered}
$$

and

$$
t_{1}=t_{1}(r, \xi, \bar{\xi})=t_{0}(r)-\frac{2}{3} d t_{0} / d r(\partial \log P / \partial r)^{-1}
$$

The density is given by

$$
\varrho=\frac{1}{6 \pi G\left(t-t_{0}\right)\left(t-t_{1}\right)}
$$


The 2-metric $d \xi d \bar{\xi} / P^{2}$ is that of a sphere of radius $R$ which may, by a coordinate transformation $r^{\prime}=f(r)$, be set equal to $r$ in any region where $d R / d r \neq 0$. The metric reduces to the familiar $k=0$ Bondi-Tolman metric in the case of spherical symmetry:

$$
\begin{gathered}
d s^{2}=d t^{2}-\left(t-t_{0}\right)^{-2 / 3}\left(t-t_{1}\right)^{2} d r^{2}-\left(t-t_{0}\right)^{4 / 3} d \xi d \bar{\xi} /\left[\frac{1}{2}(1+\xi \bar{\xi})\right]^{2}, \\
t_{0}=t_{0}(r), \quad t_{1}=t_{1}(r)=t_{0}(r)+\frac{2}{3} r d t_{0} / d r .
\end{gathered}
$$

\section{The Case $\boldsymbol{\beta}^{\prime}=\mathbf{0}, \dot{\boldsymbol{\beta}}_{\xi}=0$}

Under these assumptions we have again that Eq. (20) holds but now with the variable $r$ supressed:

$$
\beta=\psi(t)+v(\xi, \bar{\xi})
$$

Eqs. (10), (12), and (12') are all automatically satisfied, and Eqs. (11), (11') imply

$$
\alpha=\log (\mu(t, r)+\phi(t) \sigma(r, \xi, \bar{\xi}))
$$

where $\phi=e^{\psi}$. As in the previous section we have from (14) that

$$
4 e^{-2 v} v_{\xi \xi}=e^{2 \psi}\left(2 \ddot{\psi}+3 \dot{\psi}^{2}\right)=-k, \quad k=\text { const. }
$$

Hence

$$
e^{\psi}=\phi=F\left(k, l ; t-t_{0}\right), \quad k, l, t_{0}=\text { consts. }
$$

$\psi$ and $v$ may be defined in such a way that $k= \pm 1$ or 0 , and a coordinate transformation $t \rightarrow t-t_{0}$ may be used to set $t_{0}=0$. Again we have

$$
e^{-v}=a \xi \bar{\xi}+B \xi+\bar{B} \bar{\xi}+c
$$

where $a, c$ are real constants, $B a$ complex constant satisfying

$$
a c-B \bar{B}=\frac{1}{4} k \text {. }
$$

From Eqs. (13), (13') we now find that

$$
\left(e^{-v} \sigma\right)_{\xi \xi}=\left(e^{-v} \sigma\right)_{\bar{\xi} \bar{\xi}}=0
$$

whence

$$
\sigma=[u(r) \xi \bar{\xi}+V(r) \xi+\bar{V}(r) \bar{\xi}+w(r)] /(a \xi \bar{\xi}+B \xi+\bar{B} \bar{\xi}+c)
$$

where $u, w$ are real functions and $V$ a complex function of $r$.

Substitution in Eq. (15) results in

where

$$
\phi \ddot{\mu}+\dot{\phi} \dot{\mu}=\ddot{\phi} \mu=f(r)
$$

$$
f(r)=2 e^{-2 v} \sigma_{\xi \bar{\xi}}+k \sigma=2[c u(r)+a w(r)-B \bar{V}(r)-\bar{B} V(r)] .
$$


These metrics are generalizations of the Kantowski-Sachs homogeneous and anisotropic models [4] which arise when we put $k= \pm 1, \sigma=f=0, \mu=\mu(t)$. Using Eq. (9) the various cases may, after suitable coordinate transformations, be brought to the following canoical forms:

(i) $k=+1, l>0 ; \quad e^{-v}=\frac{1}{2}(1+\xi \bar{\xi})$,

$$
\begin{gathered}
\sigma=[u(r) \xi \bar{\xi}+V(r) \xi+\bar{V}(r) \bar{\xi}+w(r)] / \frac{1}{2}(1+\xi \bar{\xi}), \\
\phi=F(1, l ; t)=l \cos ^{2} \eta, \quad t=l\left(\eta+\frac{1}{2} \sin 2 \eta\right), \\
\mu=\varepsilon+(\varepsilon \eta+b(r)) \tan \eta, \quad \varepsilon= \pm 1,0, \\
\kappa \varrho=(\varepsilon+3 l \sigma) l^{2} \cos ^{2} \eta(\mu+\phi \sigma) .
\end{gathered}
$$

(ii) $k=-1 ; \quad e^{-v}=\frac{1}{2}(\xi \bar{\xi}-1)$,

$$
\sigma=[u(r) \xi \bar{\xi}+V(r) \xi+\bar{V}(r) \bar{\xi}+w(r)] / \frac{1}{2}(\xi \bar{\xi}-1),
$$

(iia) $l<0 ; \quad \phi=F(-1, l ; t)=-l \cosh ^{2} \eta, t=-l\left(\eta+\frac{1}{2} \sinh 2 \eta\right)$,

$$
\begin{gathered}
\mu=\varepsilon-(\varepsilon \eta+b(r)) \tanh \eta, \quad \varepsilon= \pm 1,0, \\
\kappa \varrho=(-\varepsilon+3 l \sigma) / l^{2} \cosh ^{2} \eta(\mu+\phi \sigma),
\end{gathered}
$$

(iib) $l>0 ; \phi=F(-1, l ; t)=l \sinh ^{2} \eta, \quad t=l\left(-\eta+\frac{1}{2} \sinh 2 \eta\right)$,

$$
\begin{aligned}
\mu & =\varepsilon-(\varepsilon \eta+b(r)) \operatorname{coth} \eta, \quad \varepsilon= \pm 1,0, \\
\kappa \varrho & =(-\varepsilon+3 l \sigma) / l^{2} \sinh ^{2} \eta(\mu+\phi \sigma),
\end{aligned}
$$

(iic) $l=0 ; \phi=F(-1,0 ; t)=t$,

$$
\begin{aligned}
\mu & =\varepsilon+b(r) \log t, \quad \varepsilon= \pm 1,0, \\
\kappa \varrho & =2 b(r) / t^{2}(\mu+\phi \sigma) .
\end{aligned}
$$

(iii) $k=0 ; e^{-v}=1$,

$$
\sigma=u(r) \xi \bar{\xi}+V(r) \xi+\bar{V}(r) \bar{\xi}+w(r),
$$

(iiia) $l>0 ; \phi=F(0, l ; t)=t^{2 / 3}, \quad$ (i.e. can set $l=4 / 9$ )

$$
\begin{aligned}
\mu & =\varepsilon t^{-1 / 3}+\frac{9}{5} u(r) t^{4 / 3}, \quad \varepsilon= \pm 1.0, \\
& =\frac{4}{9}\left(4 \varepsilon t^{-1 / 3}+3 t^{2 / 3} \sigma\right) / t^{2}(\mu+\phi \sigma),
\end{aligned}
$$

(iiib) $l=0 ; \phi=1$,

$$
\begin{aligned}
\mu & =\varepsilon t+u(r) t, \quad \varepsilon= \pm 1,0, \\
\kappa \varrho & =-4 u(r) /(\mu+\phi \sigma) .
\end{aligned}
$$

Vacuum solutions can only arise as follows. In cases (i), (ii a), and (iib) we must have $\varepsilon=0, \sigma=0$, and the solutions are various relatives of the Schwarzschild solution (see [4]). In cases (ii b); (iii b) all vacuum solutions have vanishing Reimann tensor and are therefore flat, while in (iiia) there is no vacuum solution, for $\varrho=0$ implies $\varepsilon=\sigma=0$ and this gives rise to a singular metric. 


\section{Case $\dot{\boldsymbol{\beta}}_{\xi} \neq 0$}

In this case there is no loss of generality in assuming $\alpha=\alpha(t, y), \beta=\beta(t, y)$, where $y=\frac{1}{2}(\xi+\bar{\xi})$.

Proof. From Eq. (19) we see at once that we must have $\beta^{\prime}=0$. On the other hand, from $\partial / \partial r$ of (18) we now obtain $\alpha_{\xi}^{\prime}=0$. But from Eq. (11) we see that $\alpha_{\xi} \neq 0$, whence from $\partial / \partial r$ of (11) we have $\dot{\alpha}^{\prime}=0$. Hence $\alpha=f(r)+g(t, \xi, \bar{\xi})$, and after a coordinate transformation of the form $r=h\left(r^{\prime}\right)$ it is possible to set $f(r)=0$. Thus we may assume

$$
\alpha=\alpha(t, \xi, \bar{\xi}), \quad \beta=\beta(t, \xi, \bar{\xi}) .
$$

Eq. (17) may be written

whence

$$
\begin{gathered}
(\partial / \partial t)\left(\alpha_{\xi} / \alpha_{\bar{\xi}}\right)=0, \\
\alpha_{\xi}=e^{i \lambda(\xi, \bar{\xi})} \alpha_{\xi} .
\end{gathered}
$$

Let $\sigma(\xi, \bar{\xi})$ be any non-zero real solution of the differential equation

$$
\sigma_{\xi}=e^{i \lambda} \sigma_{\bar{\xi}}, \quad\left(e . g . \sigma=\alpha\left(t_{0}, \xi, \bar{\xi}\right)\right),
$$

then it follows that

$$
\alpha=\alpha(t, \sigma) .
$$

Now from (13), (13') we have

whence

$$
\beta=\frac{1}{2}\left(\alpha+\log \alpha_{\xi}+\log \bar{f}(t, \bar{\xi})\right)=\frac{1}{2}\left(\alpha+\log \alpha_{\bar{\xi}}+\log f(t, \xi)\right),
$$

$$
e^{i \lambda}=f(t, \xi) / \bar{f}(t, \bar{\xi})=f\left(t_{0}, \xi\right) / \bar{f}\left(t_{0}, \bar{\xi}\right),
$$

where $t_{0}=$ const. Define the complex function $g(\xi)$ from the differential equation

$$
d g / d \xi=f\left(t_{0}, \xi\right),
$$

and writting $\sigma=\sigma(g(\xi), \bar{g}(\bar{\xi}))$, we see that

$$
\partial \sigma / \partial g=\partial \sigma / \partial \bar{g}
$$

so that $\sigma=\sigma(g+\bar{g})$. After performing a coordinate transformation $\xi \rightarrow \xi^{\prime}=2 g(\xi)$, it is clear that we may assume

and hence that

$$
\alpha=\alpha(t, y), \quad y=\frac{1}{2}(\xi+\bar{\xi}),
$$

$$
\alpha_{\xi}=\alpha_{\bar{\xi}} .
$$

Substituting into Eqs. (13), (13') gives at once that $\beta_{\xi}=\beta_{\bar{\xi}}$, whence

$$
\beta=\beta(t, y) .
$$

Thus in this case there is a two-parameter group of motions with commuting spacelike Killing vectors (plane symmetry). The particular form of the metric discussed here is a rather unnatural restriction with respect to these symmetries (the general case of vacuum metrics possessing these symmetries have already been dealt with elsewhere [8]). However for the sake of completeness one may write out the equations resulting from imposing these restrictions and integrate 
them. A tedious and uninstructive computation reveals that in fact there are no solutions to these equations, so that the models discussed in Sections 4 and 5 are all the irrotational dust models with the metric form (1).

\section{Appendix \\ 2-Surfaces of Constant Curvature}

Consider a 2-metric expressed in complex conformal coordinates

$$
d \sigma^{2}=e^{2 v} d \xi d \bar{\xi}, \quad v=v(\xi, \bar{\xi})
$$

The Gaussian curvature is given by

$$
K=-4 e^{-2 v} v_{\xi \xi} .
$$

If $K=$ const., we find immediately from (A2) that

whence

$$
\left(v_{\xi \xi}-\left(v_{\xi}\right)^{2}\right)_{\bar{\xi}}=0,
$$

$$
v_{\xi \xi}-\left(v_{\xi}\right)^{2}=\tau(\xi) \text {. }
$$

Any coordinate transformation of the form $\xi=f\left(\xi^{\prime}\right), \bar{\xi}=\bar{f}\left(\bar{\xi}^{\prime}\right)$ will preserve the conformal form (A1) of the metric, but with a new function

$$
v^{\prime}\left(\xi^{\prime}, \bar{\xi}^{\prime}\right)=v(\xi, \bar{\xi})+\frac{1}{2} \log f_{\xi^{\prime}}+\frac{1}{2} \log \bar{f}_{\bar{\xi}^{\prime}} .
$$

Hence the function $\tau$ in (A3) undergoes the transformation

$$
\tau^{\prime}\left(\xi^{\prime}\right)=\tau(\xi)\left(f_{\xi^{\prime}}\right)^{2}+\frac{1}{2}\left(\log f_{\xi^{\prime}}\right)_{\xi^{\prime} \xi^{\prime}}-\frac{1}{4}\left(\log f_{\xi^{\prime}}\right)_{\xi^{\prime}},
$$

and such a transformation may clearly be chosen such that $\tau^{\prime}\left(\xi^{\prime}\right)=0$. Thus without loss of generality we may take

whence

$$
\begin{aligned}
\left(e^{-v}\right)_{\xi \xi} & =e^{-v}\left(-v_{\xi \xi}+\left(v_{\xi}\right)^{2}\right)=0, \\
e^{-v} & =a \xi \bar{\xi}+B \xi+\bar{B} \bar{\xi}+c,
\end{aligned}
$$

and substitution in (A2) implies

$$
a c-B \bar{B}=K / 4 .
$$

A further bilinear transformation

$$
\xi=\frac{P \xi^{\prime}+Q}{R \xi^{\prime}+S}
$$

may be applied to reduce (A4) to a canonical form

$$
e^{-v}=\frac{1}{2}(1+K \xi \bar{\xi})
$$

$K=+1$ is the unit sphere, $K=0$ is flat space, and $K=-1$ is the unit pseudo-sphere. 


\section{References}

1. Heckmann, O., Schücking, E.: In: Witten, L. (Ed.): Graviation, an introduction to current research. New York: Wiley 1962

2. Eardley, D., Liang, E., Sachs, R.: J. Math. Phys. 13, 99 (1972)

3. Bondi,H.: Monthly Notices Roy. Astron. Soc. 410, 107 (1947)

4. Kantowski, R., Sachs, R. K.: J. Math. Phys. 7, 443 (1966)

5. Raychaudhuri, A. K.: Phys. Rev. 98, 1123 (1955)

6. Szekeres, P.: Nuovo Cimento, 17 B, 187 (1973)

7. Ehlers, J., Kundt, W.: In Ref. 1

8. Szekeres, P.: J. Math. Phys. 13, 286 (1972)

Communicated by J. Ehlers

P. Szekeres

Department of Mathematical Physics The University of Adelaide

G.P.O. Box 488

Adelaide, South Australia 5001 